\title{
RNA interference-mediated silencing of genes involved in the immune responses of the soybean pod borer Leguminivora glycinivorella (Lepidoptera: Olethreutidae)
}

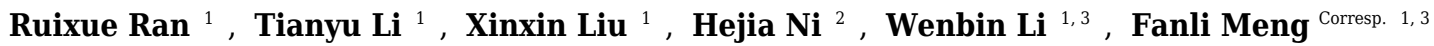 \\ ${ }^{1}$ Key Laboratory of Soybean Biology in the Chinese Ministry of Education, Northeast Agricultural University, Harbin, Heilongjiang, China \\ 2 Colleges of Life Science, Northeast Agricultural University, Harbin, Heilongjiang, China \\ 3 Key Laboratory of Biology and Genetics \& Breeding for Soybean in Northeast China, Ministry of Agriculture, Northeast Agricultural University, Harbin, \\ Heilongjiang, China
}

Corresponding Author: Fanli Meng

Email address: mengfanli@neau.edu.cn

RNA interference (RNAi) technology may be useful for developing new crop protection strategies against the soybean pod borer (SPB; Leguminivora glycinivorella), which is a critical soybean pest in northeastern Asia. Immune-related genes have been recently identified as potential RNAi targets for controlling insects. However, little is known about these genes or mechanisms underlying their expression in the SPB. In this study, we completed a transcriptome-wide analysis of SPB immune-related genes. We identified 41 genes associated with SPB microbial recognition proteins, immune-related effectors or signalling molecules in immune response pathways (e.g., Toll and immune deficiency pathways). Eleven of these genes were selected for a double-stranded RNA artificial feeding assay. The down-regulated expression levels of LgToll-5-1a and LgPGRP-LB2a resulted in relatively high larval mortality rates and abnormal development. Our data represent a comprehensive genetic resource for immune-related SPB genes, and may contribute to the elucidation of the mechanism regulating innate immunity in Lepidoptera species. Furthermore, two immune-related SPB genes were identified as potential RNAi targets, which may be used in the development of RNAi-mediated SPB control methods. 
1 Running title: Immune -related genes of Leguminivora glycinivorella

2 RNA interference-mediated silencing of genes involved in the immune responses of the

3 soybean pod borer Leguminivora glycinivorella (Lepidoptera: Olethreutidae)

4 Ruixue Ran ${ }^{1}$, Tianyu Li ${ }^{1}$, Xinxin Liu ${ }^{1}, \mathrm{HejiaNi}^{2}$, Wenbin Li ${ }^{1,3}$, Fanli Meng 1,3*

$5 \quad{ }^{1}$ Key Laboratory of Soybean Biology in the Chinese Ministry of Education, Northeast

6 Agricultural University, Harbin 150030, China

$7 \quad{ }^{2}$ Colleges of Life Science, Northeast Agricultural University, Harbin, China, 150030

$8 \quad{ }^{3}$ Division of Soybean Breeding and Seeds, Soybean Research \& Development Center, CARS

9 (Key Laboratory of Biology and Genetics \& Breeding for Soybean in Northeast China, Ministry

10 of Agriculture), Northeast Agricultural University, Harbin 150030, China

12 Ruixue Ran: ranruixue@outlook.com

13 Tianyu Li: litianyu151243@outlook.com

14 Xinxin Liu: Liu_xinxin6@163.com

15 HejiaNi:nhjwinner@163.com

16 Wenbin Li: wenbinli@neau.edu.cn

17 Fanli Meng: mengfanli@neau.edu 
18 Ruixue Ran and Tianyu Li contributed equally to this work

$19{ }^{*}$ Corresponding author:

20 Fanli Meng

21 Key Laboratory of Soybean Biology in the Chinese Ministry of Education, Northeast Agricultural

22 University, Harbin, China, 150030

23 Email: mengfanli@neau.edu

24 Phone: (+86) 451-55191413

25 Fax: (+86) 451-55103336

26

27

28

29

30

31

32

33

34

35

36

37

38

39

40

41

42

43 


\section{Abstract}

51 RNA interference (RNAi) technology may be useful for developing new crop protection strategies

52 against the soybean pod borer (SPB; Leguminivora glycinivorella), which is a critical soybean pest

53 in northeastern Asia. Immune-related genes have been recently identified as potential RNAi targets

54 for controlling insects. However, little is known about these genes or mechanisms underlying their

55 expression in the SPB. In this study, we completed a transcriptome-wide analysis of SPB immune-

56 related genes. We identified 41 genes associated with SPB microbial recognition proteins,

57 immune-related effectors or signalling molecules in immune response pathways (e.g., Toll and

58 immune deficiency pathways). Eleven of these genes were selected for a double-stranded RNA

59 artificial feeding assay. The down-regulated expression levels of $L g$ Toll-5-1a and $\operatorname{Lg} P G R P-L B 2 a$

60 resulted in relatively high larval mortality rates and abnormal development. Our data represent a

61 comprehensive genetic resource for immune-related SPB genes, and may contribute to the

62 elucidation of the mechanism regulating innate immunity in Lepidoptera species. Furthermore,

63 two immune-related SPB genes were identified as potential RNAi targets, which may be used in 
64 the development of RNAi-mediated SPB control methods.

\section{INTRODUCTION}

66 Leguminivora glycinivorella (Mats.) obraztsov [soybean pod borer (SPB)] belongs to the

67 order Lepidoptera and family Olethreutidae. The SPB is the major pest of soybean in northeastern

68 Asia (Zhao et al., 2008; Meng et al., 2017a). The larvae use the immature beans as a food source

69 until they reach maturity, resulting in soybean yield losses of up to $40 \%$ (Meng et al., 2017b).

70 Insecticides have been used to control SPB infestations over the past three decades. However,

71 larvae within soybean pods that are under a closed canopy are often not exposed to the applied

72 insecticides. Because of the lack of effective SPB-resistant germplasm, conventional breeding has

73 not resulted in the production of new SPB-resistant cultivars. Therefore, the SPB remains a major

74 pest and is responsible for substantial soybean yield losses (Wang et al., 2014; Song et al., 2015).

75 Consequently, soybean breeders and growers are interested in developing new strategies for

76 controlling SPB infestations, with RNA interference (RNAi) representing a promising option

77 (Khajuria et al., 2015; Fishilevich et al., 2016).

78 RNAi involves the degradation of specific endogenous mRNAs by homologous double-stranded

79 RNAs (dsRNAs) (Fire et al., 1998). Depending on the function of the targeted gene, RNAi can

80 inhibit insect growth or result in death (Joga et al., 2016; Christiaens et al., 2014). RNAi is 
81 conserved in nearly all eukaryotic organisms, and feeding insect pests dsRNA molecules may be

82 useful in protecting agriculturally important crops (feeding RNAi or plant-mediated RNAi) (Mao

83 \& Zeng, 2014; Ulrich et al., 2015). However, microinjection RNAi is particularly successful when

84 targeting genes involved in immune responses (Terenius et al., 2011). The effectiveness of the

85 RNAi technique for controlling pests depends on whether appropriate candidate genes are targeted

86 because RNAi efficacy and the RNAi signal transmission vary among genes (Huvenne \&

87 Smagghe, 2010). Whileapplying RNAi technology to control Lepidoptera insects (i.e., moths

88 and butterflies) has been problematic (Shukla et al., 2016), the information available regarding its

89 efficiency has recently increased. Insects such as Drosophila melanogaster and Bombyx mori have a vigorous innate immune

91 systems with which to defend against microbial infections (Myllymäki et al., 2014; Parsons et al.,

92 2016; Yang et al., 2017; Chen et al., 2018). Peptidoglycan recognition proteins (PGRPs) are

93 important pattern recognition receptors that detect peptidoglycan (PGN) in the cell walls of gram-

94 negative and gram-positive bacteria. PGRPs activate the Toll or immune deficiency (IMD)/JNK

95 pathways or induce proteolytic cascades that generate antimicrobial peptides (Gao et al., 2015;

96 Chen et al., 2014). Antimicrobial peptides are critical for defending against invading pathogens

97 and for protecting insects against infections (Gegner et al., 2018). However, little is known about 
98 SPB immune-related genes or the associated immune responses.

99 To identify the immune-related genes of SPB, we generated SPB transcriptome datasets

100 based on Illumina sequencing. These datasets were used to identify many genes associated with

101 microbe recognition, immune-related signalling, and defence effectors. Furthermore, RNAi was

102 applied to study the effects of silencing immune-related genes on first instar larvae. A feeding

103 assay involving an artificial diet supplemented with dsRNA was used to identify candidate target

104 genes for controlling the SPB by RNAi.

105 MATERIALS\& METHODS

106 Insect rearing

107 L. glycinivorella eggs collected from a naturally infested soybean field at the experimental

108 station of Northeast Agricultural University in Harbin, China were hatched at $26^{\circ} \mathrm{C}$. The resulting

109 larvae were reared on an artificial diet prepared in our laboratory (Meng et al., 2017a). Adult moths

110 were fed a $5 \%$ honey solution, and were allowed to oviposit on young bean pods. The first instar

111 larvae were selected and subjected to artificial diet feeding experiments.

112 Illumina sequencing

113 The T3 dsSpbP0 (double-stranded SBP ribosomal protein P0 RNAi transgenic soybean line)

114 and wild-type 'DN50' plants, provided by the Key Laboratory of Soybean Biology of the Chinese 
115 Education Ministry, Harbin, China, were grown in a greenhouse at $24 \pm 1{ }^{\circ} \mathrm{C}$ with $60 \%$ relative

116 humidity under a 16-h light/8-h dark cycle (Meng et al., 2017b). At the R5 soybean stage (fully

117 developed pods), three replicates of 50 first-instar larvae were reared on soybean pods of DN50

118 and T3 dsSpbP0 plants. All larvae were collected after $3 \mathrm{~d}$ and used to construct cDNA library.

119 cDNA library preparation and Illumina sequencing were conducted by the Biomarker Technology

120 Company (Beijing, China). Briefly, total RNA was extracted from six pooled larvae using TRIzol

121 reagent (Invitrogen, Carlsbad, CA, USA). The first-strand cDNA was synthesised using random

122 hexamer-primers from purified Poly (A) mRNA. Second-strand cDNA was synthesised using

123 DNA polymerase I and RNaseH, and then purified using a QiaQuick PCR extraction kit (Qiagen,

124 Hilden, Germany). cDNA fragments of a suitable length (300-500 bp) were obtained by agarose

125 gel electrophoresis and amplified by PCR to construct the final cDNA libraries using NEB Next

126 Ultra RNA Library Prep Kit for Illumina (NEB, Ipswich, MA,USA). The cDNA library was

127 sequenced on Illumina HiSeq 2000 system. The unigenes from six samples were combined to

128 create the SPB unigene database (Chen et al., 2014; Meng et al., 2017c). All raw transcriptome

129 data have been deposited in the NIH Short Read Archive (accession numbers SRR5985984-

130 SRR5985989).

131 Identification of immune-related genes 
132 A list of immune-related genes was compiled based on the available relevant literature (Table

133 S1), and homologous B. mori, D. melanogaster, Danaus plexippus and Papilio polytes genes in

134 the GenBank database were identified (Guan \& Mariuzza, 2007; Xu et al., 2012). The tBLASTn

135 algorithm-based tool was used to complete sequence similarity searches of the SPB transcriptome

136 database (Boratyn et al., 2013). The 41 SPB immune-related genes were submitted

137 to NCBI GenBank and their Accession numbers are shown in TableS1.

138 Phylogenetic and domain analyses

139 Amino acid sequences were aligned with the Multiple Alignment program clustal omega

140 (https://www.ebi.ac.uk/Tools/msa/clustalo/), and the phylogenetic tree was constructed in MEGA

1415 based on the neighbour-joining method with 1000 bootstrap replicates (Tamura et al., 2011;

142 Sievers et al., 2011). The architecture of the protein domains was analysed using the SMART

143 program (http://smart.embl-heidelberg.de/).

144 dsRNA synthesis

145 We synthesised dsRNAs using the T7 RiboMAX Express Large Scale RNA Production

146 System (Promega, Madison, WI, USA). The T7 RNA polymerase promoter sequence was added

147 to each end of the DNA templates during PCR amplifications. The primers containing the T7 RNA

148 polymerase promoter were designed using Primer-BLAST 
149 (https://www.ncbi.nlm.nih.gov/tools/primer-blast/) (Table S2). For the negative control, the green

150 fluorescent protein $(G F P)$ gene was amplified from the PCAMBIA1302 vector as a template for

151 GFP dsRNA synthesis. The template DNA and single-stranded RNA were eliminated from the

152 transcription reaction by DNase I and RNase A, respectively. The prepared dsRNAs were purified

153 by a phenol/chloroform extraction followed by an ammonium acetate precipitation. The dsRNAs

154 were ultimately suspended in ultrapure water and quantified using a Nano Drop 2000

155 spectrophotometer (Thermo Scientific, Waltham, MA, USA).

156 SPB feeding bioassay

157 The first-instar SPB larvae were fed an artificial diet containing dsRNA $(10 \mu \mathrm{g} / \mathrm{g})$ for specific

158 target genes as described by Meng et al. (2017a). Control larvae were treated with the same

159 concentration of GFP dsRNA. The feeding bioassay was completed in triplicate with 50 larvae per

160 treatment or control. Three biological replicates were used for each treatment. The larvae were

161 reared for $15 \mathrm{~d}$ at $26^{\circ} \mathrm{C}$ under a $16-\mathrm{h}$ light/ 8 -h dark cycle with $65 \%$ relative humidity. The dsRNA-

162 supplemented artificial diet was refreshed every $3 \mathrm{~d}$. Body weight, mortality, and phenotypic

163 abnormalities were recorded every $3 \mathrm{~d}$.

164 Quantitative real-time PCR $(q R T-P C R)$

165 For every treatment, two surviving larvae were randomly collected at each time point from 
166 all of the biological replicates from 0 to $15 \mathrm{~d}$ after larvae were fed the artificial diet containing the

167 target gene's dsRNA, frozen in liquid nitrogen and stored at $-80^{\circ} \mathrm{C}$. Total RNA was extracted from

168 two pooled larvae using the RNApure Tissue Kit (DNase I) (CWBIO, Beijing, China).

169 Additionally, primer sets were synthesised (Table S2). The extracted RNA samples were treated

170 with DNase I (Invitrogen) to remove any contaminating genomic DNA. They were then used as

171 the template for first-strand cDNA synthesis with the TIANScript RT Kit (Tiangen, Beijing,

172 China). The qRT-PCR analysis was completed using the SYBR Green kit (Bio-Rad, Hercules, CA,

173 USA) and a Roche LightCycler ${ }^{\circledR} 480$ real-time PCR system (Roche, Basel, Switzerland). Each

174 reaction used cDNA corresponding to $50 \mathrm{ng}$ of total RNA and each primer at a final concentration

175 of $100 \mathrm{~nm}$ in $20 \mu \mathrm{l}$ reaction. Controls included non-RT controls (50ng total RNA without reverse

176 transcription was used to detect genomic DNA contamination) and non-template controls (water

177 template).The qRT-PCR conditions were as follows: $95^{\circ} \mathrm{C}$ for $5 \mathrm{~min} ; 40$ cycles at $95^{\circ} \mathrm{C}$ for $30 \mathrm{~s}$,

$17860^{\circ} \mathrm{C}$ for $15 \mathrm{~s}$, and $72^{\circ} \mathrm{C}$ for $45 \mathrm{~s} ; 95^{\circ} \mathrm{C}$ for $1 \mathrm{~min}$ and $55^{\circ} \mathrm{C}$ for $1 \mathrm{~min}$. At the end of each qRT-

179 PCR experiment, a melt curve was generated to check for primer-dimer formation. The efficiencies

180 of the qRT-PCR primer pairs were greater than 90\% (Table S2). The qRT-PCR analysis contained

181 three biological replicates, each having three technical replicates (Meng et al., 2017c). Each target

182 gene's qRT-PCR products were sequenced to confirm their identities. 
183 Relative expression levels were calculated by the following formula using LightCycler 480

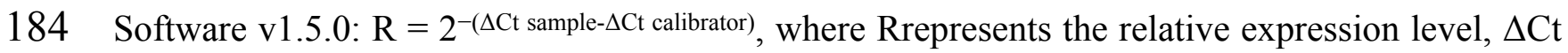

185 sample is the average difference between the $\mathrm{Ct}$ of the gene and that of SBPß-actin in the

186 expreimental sample (Meng et al., 2017b), and $\Delta \mathrm{Ct}$ calibrator is the average difference between

187 the $\mathrm{Ct}$ of the gene and the e SBPß-actin in the calibrator. A representative sample was set as the

188 calibrator (Bustin et al., 2009). The differences in Ct values between technical replicates was less

189 than 0.5 . The relative expression level of $0 \mathrm{~d}$ for each target gene was set as a benchmark. The

190 relative expression level of other time point for each target gene was used to compare with 0d

191 value.

192 Statistical analyses

193 All of the data in this study are presented as mean \pm SE. Significant differences were

194 determined by one-way analysis of variance followed by least significant difference tests for mean

195 comparisons. The statistical analysis was performed with SAS 9.21 software (SAS Institute, Cary,

196 NC, USA). $P$-values were corrected by Bonferroni.

197 RESULTS

198 Identification of Leguminivora glycinivorella immune-related genes

199 Amino acid sequences encoded by D. melanogaster, B. mori, and Manduca sexta

200 (Lepidoptera) immune-related genes were used to search SPB transcriptome sequences. The 41 
201 identified putative SPB immune-related genes were functionally classified into three groups,

202 microbial recognition, immune signalling, and immune effector molecules (Table S1).

203 Microbial recognition molecules

204 The PGRPs recognize conserved molecular patterns present in pathogens, but absent in the

205 host, including PGNs, which are essential cell wall components of almost all bacteria. The PGRPs

206 are encoded by a highly conserved gene family in insects, and are generally classified into two

207 types (short and long) (Dziarski \& Gupta, 2006; Yang et al., 2017). We identified eight SPB

208 PGRPs, four short and four long types, with similarities to D. melanogaster PGRP-SC, PGRP-SD,

209 and PGRP-LB (Fig. 1). Five (LgPGRP-SC1a, LgPGRP-SC1b, LgPGRP-SD1b, LgPGRP-LB1,

210 and LgPGRP-LB2b) of the eight identified PGRPs were predicted to be secreted proteins, based

211 on the presence of putative signal peptides, that function as amidases. LgPGRP-LB lacks a putative

212 signal peptide, but contains a transmembrane region and amidase domain, suggesting that it serves

213 as a transmembrane PGN receptor. In contrast, LgPGRP-SD1a and LgPGRP-LB2a carry only the

214 PGRP domain, implying that they are intracellular proteins (Table S1).

215 The gram-negative bacteria-binding proteins (GNBPs) and $\beta$-1,3-glucan recognition proteins

216 ( $\beta$ GRPs) belong to a subfamily of pattern recognition receptors and have strong affinities for the

217 lipopolysaccharide of gram-negative bacteria and the $\beta$-1,3-glucan of fungi, but not for the PGN 
218 of gram-positive bacteria. Of the three GNBPs produced by D. melanogaster (GNBP1, GNBP2,

219 and GNBP3), GNBP1 interacts with PGRP-SA to form a hydrolytic complex that activates the

220 Toll pathway in response to gram-positive bacteria, while GNBP3 is required for detecting fungi

221 and activating the Toll pathway (Hughes, 2012; Rao et al., 2017). We identified one GNBP gene

222 in the SPB transcriptome datasets. The neighbour-joining phylogenetic analysis indicated that

$223 \operatorname{Lg} G N B P 3$ is an ortholog of $D m G N B P 3$ (Fig. S1). A comparison between the deduced amino acid

224 sequences and the D. melanogaster GNBP sequences indicated that LgGNBP3 contains a putative

225 N-terminal $\beta$-1,3-glucan-recognition domain (CBM39) and a C-terminal glucanase-like domain

226 (glycosyl hydrolase family 16), suggesting that LgGNBP3 may bind to fungal $\beta$-1,3-glucan.

227 Immune signalling molecules

228 After specific ligands are detected, microbial recognition molecules activate or modulate

229 various immune response pathways. Here, we identified genes associated with the Toll and IMD

230 pathways, which are the major signalling pathways that mediate the innate immunity of insects.

231 The Toll pathway regulates the production of antimicrobial peptides in response to infections by

232 fungi or gram-positive bacteria with lysine-type PGNs in their cell walls (Roh et al., 2009). The

233 Toll receptor, which is responsible for the signal transduction associated with the Toll pathway, is

234 vital for insect innate immune responses and embryo development (Takeda \& Akira, 2004; Benton 
235 et al., 2016). In this study, we identified 10 genes encoding Toll receptors in the SPB transcriptome

236 datasets. The TIR domain is highly conserved in insect Toll families. To investigate the

237 orthologous relationships among these genes, we constructed a phylogenetic tree based on an

238 alignment of the TIR domains from all SPB and D. melanogaster Toll proteins. The Toll receptors

239 analysed in this study formed six major clusters, namely Toll-3, Toll-4, Toll-5, Toll-6, Toll-7 and

240 Toll-8 (Fig. 2A). Based on the phylogenetic tree, the L. glycinivorella Toll genes were designated

241 as LgTLR-3, LgTLR-4, LgToll-5-1a, LgToll-5-1b, LgToll-6, TLR-6-1, TLR-6-2, LgToll-7, LgTLR-

2427 and LgToll-8. All 10 predicted proteins contain an extracellular LRR domain as well as

243 transmembrane and cytoplasmic TIR domains (Fig. 2B). We also identified sequences matching

244 the intracellular components, ECSIT and Tollip, which affect the Toll signalling pathway (Table

245 S1).

246 The IMD pathway is mainly activated by gram-negative bacterial infections. Additionally,

247 IMD signal transduction is reportedly mediated by IMD, fas-associated death domain protein

248 (FADD), death-related ced-3/Nedd2-like caspase (Dredd), inhibitor of apoptosis protein 2 (IAP2),

249 transforming growth factor $\beta$-activated kinase (TAK1), TAK1-binding 2 (Tab2), ubiquitin

250 conjugating 13 (Ubc13), and an inhibitor of nuclease factor B kinase subunits $\mathrm{b}$ and $\mathrm{g}$ (IKKb and

251 IKKg)( Bao et al., 2013; Myllymäki et al., 2014). Of these,we only identified sequences that were 
252 homologous to FADD, Dredd, and IAP2 (Table S1).

\section{Immune-related effector genes}

254 The PGRPs and $\beta$ GRPs detect PGNs and $\beta$-1,3-glucans, which activates a clip-domain

255 serine protease (CLIP) cascade that converts prophenoloxidase to active phenoloxidase, leading

256 to the melanisation responses involved in eliminating pathogens(Monwan, Amparyup \&

257 Tassanakajon, 2017; Li et al., 2016). We identified two CLIP genes (LgSnake-1 and LgSnake-2)

258 in the SPB transcriptome datasets. The deduced amino acid sequences each contain a clip

259 domain at the N-terminus and a serine protease domain at the C-terminus (Fig. S2). Serine

260 protease inhibitors (i.e., serpins) negatively regulate prophenoloxidase activation, which prevents

261 the excessive activation of the CLIP cascade. In this study, we identified three serpin genes,

262 Lgserpin1, Lgserpin2, and Lgserpin3, in the SPB transcriptome datasets. Their deduced amino

263 acid sequences each contained a putative signal peptide sequence and a core serpin domain,

264 suggesting that they are secreted proteins (Table S1).

265 Immune response effector genes

266 Antibacterial peptides are immune response effectors that are induced by immune

267 challenges and are important for defence responses against insects. Diverse antibacterial peptide

268 genes have been identified in many insect species, including genes encoding defensins, reeler, 
269 and lysozyme (Imler \& Bulet, 2005; Bao et al., 2011). In this study, we identified two defensin

270 genes, Lgdefensin1 and Lgdefensin2, in the SPB transcriptome datasets. The encoded amino acid

271 sequences each consisted of a putative signal peptide sequence and a core Knot1 domain (Table

272 S1). We also identified seven chicken-type (C-type) lysozymes and two invertebrate-type (I-

273 type) lysozymes in the $L$ SPB transcriptome. The C-type lysozymes are bacteriolytic enzymes

274 that hydrolyse the $\beta(1-4)$ bonds between $\mathrm{N}$-acetylglucosamine and $\mathrm{N}$-acetylmuramic acid in the

275 PGN of prokaryotic cell walls. The predicted SPB C-type proteins, with the exception of the C-

276 type 3 protein, each include an N-terminal signal peptide sequence (Table S1). Additionally, we

277 detected eight conserved cysteine residues in the L. glycinivorella C-type lysozymes (Fig. S3A)

278 as well as 12 conserved cysteine residues in the deduced SPB I-type lysozyme sequences (Fig.

279 S3B). These cysteine residues may form intramolecular disulphide bonds to enhance stability

280 and resistance against proteolytic degradation.

281 Potential RNAi targets identified in an artificial feeding assay and effects of double-stranded RNA

282 on soybean pod borer development and mortality

283 In total, 11genes representing the immune-related SPB genes were selected and analysed to

284 identify potential new RNAi targets useful for controlling the SPB. We synthesised the

285 corresponding dsRNAs in vitro and mixed them in an artificial diet. The mortality rates $3 \mathrm{~d}$ after 
286 larvae were fed artificial diets, which contained containing $10 \mu \mathrm{g} / \mathrm{g}$ dsRNA for $L g P G R P-L B$,

287 LgPGRP-LB2b, LgToll-5-1a, LgToll-5-1b, LgTLR-7, LgSerpin2 or LgChaoptin were 37\%-92\%.

288 These mortality rates were significantly greater than those of control larvae treated with GFP

289 dsRNA. Additionally, the final mortality rates of the larvae fed $\operatorname{Lg} P G R P-L B, L g P G R P-L B 2 b$,

290 LgToll-5-1a, LgSerpin 2 and LgChaoptin dsRNA were even higher at 15 d. In contrast, the artificial

291 diets containing dsRNA independently targeting $L g P G R P-L B 2 a$, LgTLR-3 and Lgitype-1 did not

292 have any statistically significant effects on larval mortality (Fig. 3). Moreover, two main

293 phenotypic differences were observed among the surviving larvae after $15 \mathrm{~d}$ of feeding. First, the

294 weights of the larvae fed LgToll-5-1b and LgItype-1 dsRNA increased more gradually than those

295 of larvae fed GFP dsRNA, and was ultimately lower after $15 \mathrm{~d}$ of feeding (Fig. 4). Additionally,

296 the LgToll-5-1b RNAi treatment resulted partly black cuticles. Second, larvae fed dsRNA targeting

$297 L g P G R P-L B$ or $L g P G R P-L B 2 a$ underwent early pupation, with pupation rates of 25 and $50 \%$,

298 respectively. The remaining larvae developed abnormally with stunted and twisted bodies (Fig. 5).

299

To investigate how larval mortality and abnormal development are correlated with the

300 relative expression levels of specific target genes, we performed qRT-PCR using total RNA

301 extracted from the surviving larvae at different time points after feeding on artificial diets. The

302 expression levels of the genes, except for those of $L g P G R P-L B 2 a, L g T L R-6-1$ and LgChaoptin 
303 decreased in larvae $3 \mathrm{~d}$ after being treated with the respective dsRNAs, while the expression levels

304 of all of the genes decreased significantly after 6 or $9 \mathrm{~d}$ (Fig. 6). Thus, the increased mortality rates

305 and abnormal development of larvae fed dsRNA may result from the down-regulated expression

306 of specific target genes. Moreover, unigenes $L g T o l l-5 a$ and $L g P G R P-L B 2 a$ may represent good

307 RNAi targets for controlling the SPB.

308 DISCUSSION

309 Insects possess efficient innate immune system that protect them from microorganisms and

310 aid in abiotic stresses (Hillyer, 2015; Parsons\& Foley, 2016). In this study, we identified 41 genes

311 in the SPB transcriptome that encode components of conserved immune signalling pathways (Toll

312 and IMD pathways) as well as pathogen recognition and immune response effectors. Most of these

313 genes contained conserved sequences that exist in orthologous D. melanogaster and B. mori genes

314 (Table S1). However, immune-related gene families have expanded or contracted in different taxa.

315 For example, the PGRP gene families in D. melanogaster, B. mori, SPB have 13, 12, and 8

316 members, respectively (Hillyer, 2015; Yang et al., 2015). In addition to sequence differences

317 among the immune-related genes, the encoded proteins exhibited diverse activities. For example,

318 four of the SPB's PGRPs are closely related to each other and form an independent cluster with

319 D. melanogaster PGRP LB (Fig. 1). Two of them contain a putative signal peptide and a conserved 
320 Ami_2 domain, while the others lack a signal peptide (Table S1). Furthermore, silencing $L g P G R P$ -

$321 L B$ and $L g$ PGRP-LB2a induced early pupation and abnormal larval development, while silencing

$322 L g P G R P-L B 2 b$ lead to significantly higher mortality rates at $3 \mathrm{~d}$ and $6 \mathrm{~d}$, indicating that $L g P G R P$ -

$323 L B 2 b$ may be essential for early larval development (Fig. 5). PGRP-LB is a catalytic amidase that

324 can degrade PGN and regulate host immune responses to infectious microorganisms by down-

325 regulating the IMD pathway (Zaidman-Rémy et al., 2006; Troll et al., 2009; Gendrin et al., 2017),

326 which protects the beneficial microbes in insects and prevents host-inflicted damage during

327 development (Hashimoto et al., 2007). In the Tsetse fly (Diptera: Glossinidae), silencing PGRP-

$328 L B$ by RNAi decreases host fecundity because of the associated cost of activating the host immune

329 response (Wang \& Aksoy, 2012).

330

The SPB is a univoltine insect. The mature larvae make cocoons in the soil, enter diapause

331 during the winter and pupate in mid-July, resulting in a diapause period of 10 months (Meng et

332 al., 2017b). In our study, $\operatorname{Lg} P G R P-L B$ and $L g P G R P-L B 2 a$ were silenced by RNAi, which broke

333 diapause and caused mature larvae to pupate. This termination of diapause result from an immune

334 response that was initiated to prevent host-inflicted damage. Further research is needed to confirm

335 that LgPGRP-LB influences the host immune responses' activation..

336 The Toll pathway is critical for innate immunity against bacteria and also affects embryonic 
337 development, olfactory neuron processes, and TNF-induced JNK-dependent cell death in $D$.

338 melanogaster (Yang et al., 2015; Valanne et al., 2011; Wu et al., 2015). Knocking down the fusilli

339 and cactin genes, which are part of the Toll pathway, is lethal for the red flour beetle (Tribolium

340 castaneum), and the silencing cactin is $100 \%$ lethal at all developmental stages (from larva to

341 adult). Additionally, the knockdown of pelle and dorsal prevents eggs from hatching in the next

342 generation (Bingsohn et al., 2017) In our study, a 10\% knocked down of LgToll-5-1a lead to

343 92\%larval mortality rate $3 \mathrm{~d}$ after feeding. While $L g T o l l-5-1 a$ is more critical for the first instar

344 larvae development. A 56\% knocked down of $L g T o l l-5-1 b$ did not impact on the survival rate of

345 larvae $3 \mathrm{~d}$ after feeding. Knocking down $\operatorname{LgToll}-5 b$ took considerably longer ( $15 \mathrm{~d}$ ) to have an

346 impact on body weights, and it prevented old cuticles from separating from larval bodies. LgToll-

347 5-1b may influence mid-to-late larval development. Thus, $L g T o l l-5$ may be play critical roles on

348 larval development, and it may function in immunity in adults. A future study will challenge Toll-

349 5-1 $a$ or LgToll-5-1b RNAi-treated insects with pathogen infections to determine their roles, if any,

350 in immunity.

351 Lysozymes are widely distributed immune effectors that exhibit muramidase activities against

352 the PGNs in bacterial cell wall to induce cell lysis (Zhou et al., 2017). In our study, the LgI-type-

3531 gene encodes a destabilase domain, which is associated with isopeptidase and antibacterial 
354 activities. The pI of LgI-type-1 is 7.93 (Table S1). Researchers have proposed that I-type

355 lysozymes with high pI values influence immunity (Kurdyumov et al., 2015; Xue et al., 2004).

356 Thus, LgI-type-1 may have isopeptidase activity and play a role in the SPB's immune system

357 CONCLUSION

358 We identified 41 genes associated with SPB microbial recognition proteins, immune-related

359 effectors, or signalling molecules of immune response pathways (e.g., Toll and immune deficiency

360 pathways). This will be useful as a comprehensive genetic resource for immune-related SPB genes

361 and may help elucidate the mechanism regulating innate immunity in Lepidoptera species. In

362 addition, the in vivo functions of 11 genes were analysed in RNAi experiments, which indicated

363 that three genes may be appropriate RNAi targets for controlling the SPB. The observations

364 described herein may be useful for future analyses of the mechanisms underlying the SPB mmune

365 response pathways and for developing RNAi-mediated methods to control SPB infestations.

366 ACKNOWLEDGEMENTS

367 We thank Professor Xiaoyun Wang (Northeast Agricultural University, China) for her technical

368 support in culturing the SPB.

369 REFERENCES

370 Bao YY, Qu LY, Zhao D, Chen LB, Jin HY, Xu LM, Cheng JA, Zhang CX. 2013. The genome-

371 and transcriptome-wide analysis of innate immunity in the brown planthopper, Nilaparvata lugens.

372 BMC genomics 14:160 DOI 10.1186/1471-2164-14-160 
373 Bao YY, Xue J, Wu WJ, Wang Y, Lv ZY, Zhang CX. 2011. An immune-induced reeler protein is

374 involved in the Bombyx mori melanization cascade. Insect Biochem Mol Biol. 41(9):696-706 DOI

375 10.1016/j.ibmb.2011.05.001

376 Benton MA, Pechmann M, Frey N, Stappert D, Conrads KH, Chen YT, Stamataki E, Pavlopoulos

377 A, Roth S. 2016. Toll Genes Have an Ancestral Role in Axis Elongation. Curr Biol. 26(12):1609-

3781615 DOI 10.1016/j.cub.2016.04.055.

379 Bingsohn L, Knorr E, Billion A, Narva KE, Vilcinskas A. 2017. Knockdown of genes in the Toll 380 pathway reveals new lethal RNA interference targets for insect pest control. Insect Mol Biol 381 26(1):92-102 DOI: 10.1111/imb.12273.

382 Boratyn GM, Camacho C, Cooper PS, Coulouris G, Fong A, Ma N, Madden TL, Matten WT, 383 McGinnis SD, Merezhuk Y, Raytselis Y, Sayers EW, Tao T, Ye J, Zaretskaya I. 2013. BLAST: a 384 more efficient report with usability improvements. Nucleic Acids Res.W29-33. doi: $38510.1093 /$ nar/gkt282.

386 Bustin SA, Benes V, Garson JA, Hellemans J, Huggett J, Kubista M, Mueller R, Nolan T, Pfaffl 387 MW, Shipley GL, Vandesompele J, Wittwer CT. 2009. The MIQE guidelines: minimum 388 information for publication of quantitative real-time PCR experiments. Clin Chem. (4):611-22. 389 doi: $10.1373 /$ clinchem.2008.112797.

390 Chen EH, Wei DD, Shen GM, Yuan GR, Bai PP, Wang JJ. 2014. De novo characterization of the

391 Dialeurodes citri transcriptome: mining genes involved in stress resistance and simple sequence 392 repeats (SSRs) discovery. Insect Mol Biol. 23(1):52-66 (2014). DOI 10.1111/imb. 12060

393 Chen K, Lu Z. 2018. Immune responses to bacterial and fungal infections in the silkworm, Bombyx 394 mori. Dev Comp Immunol. 83:3-11. doi: 10.1016/j.dci.2017.12.024. 
395 Christiaens O, Swevers L, Smagghe G. 2014. DsRNA degradation in the pea aphid (Acyrthosiphon

396 pisum) associated with lack of response in RNAi feeding and injection assay. Peptides 53:307-14

397 DOI 10.1016/j.peptides.2013.12.014

398 Dziarski R, Gupta D. 2006. The peptidoglycan recognition proteins (PGRPs). Genome Biol. 399 7(8):232 DOI 10.1186/gb-2006-7-8-232

400 Fishilevich E, Vélez AM, Khajuria C, Frey ML, Hamm RL, Wang H, Schulenberg GA, Bowling 401 AJ, Pence HE, Gandra P, Arora K, Storer NP, Narva KE, Siegfried BD. 2016. Use of chromatin 402 remodeling ATPases as RNAi targets for parental control of western corn rootworm (Diabrotica 403 virgifera virgifera) and Neotropical brown stink bug (Euschistus heros). Insect Biochem Mol Biol 404 71:58-71 DOI 10.1016/j.ibmb.2016.02.004

405 Fire A., Xu S., Montgomery M. K., Kostas S. A., Driver S. E., Mello C. C. 1998. Potent and 406 specific genetic interference by double-stranded RNA in Caenorhabditis elegans. Nature 407 391(6669):806-811 DOI 10.1038/35888.

408 Gao Y, Tang T, Gu J, Sun L, Gao X, Ma X, Wang X, Liu F, Wang J. 2015. Down regulation of 409 the Musca domestica peptidoglycan recognition protein SC (PGRP-SC) leads to overexpression 410 of antimicrobial peptides and tardy pupation. Mol Immunol 67(2 Pt B):465-74 DOI $411 \quad$ 10.1016/j.molimm.2015.08.007.

412 Gendrin M, Turlure F, Rodgers FH, Cohuet A, Morlais I, Christophides GK. 2017. The

413 Peptidoglycan Recognition Proteins PGRPLA and PGRPLB Regulate Anopheles Immunity to 414 Bacteria and Affect Infection by Plasmodium. J Innate Immun 9(4):333-342 DOI $415 \quad 10.1159 / 000452797$.

416 Gegner T, Schmidtberg H, Vogel H, Vilcinskas A. 2018. Population-specific expression of 
417 antimicrobial peptides conferring pathogen resistance in the invasive ladybird Harmonia axyridis.

418 Sci Rep. 8(1):3600. doi: 10.1038/s41598-018-21781-4.

419 Guan R, Mariuzza RA. 2007. Peptidoglycan recognition proteins of the innate immune system.

420 Trends Microbiol 15(3):127-34 DOI 10.1016/j.tim.2007.01.006

421 Hashimoto K, Mega K, Matsumoto Y, Bao Y, Yamano Y, Morishima I. 2007. Three peptidoglycan

422 recognition protein (PGRP) genes encoding potential amidase from eri-silkworm, Samia cynthia

423 ricini. Comp Biochem Physiol B Biochem Mol Biol 148(3):322-8 DOI

424 10.1016/j.cbpb.2007.06.011

425 Hillyer JF. 2015. Insect immunology and hematopoiesis. Dev Comp Immunol 58:102-18 DOI

$426 \quad 10.1016 /$ j.dci.2015

427 Hughes AL. 2012. Evolution of the $\beta G R P / G N B P / \beta-1,3$-glucanase family of insects.

428 Immunogenetics. 64(7):549-58 DOI 10.1007/s00251-012-0610-8

429 Huvenne H, Smagghe G. 2010. Mechanisms of dsRNA uptake in insects and potential of RNAi

430 for pest control: a review. J Insect Physiol 56(3):227-35 DOI 10.1016/j.jinsphys.2009

431 Imler JL, Bulet P. 2005. Antimicrobial peptides in Drosophila: structures, activities and gene

432 regulation. Chem Immunol Allergy.86:1-21 DOI 10.1159/000086648

433 Joga MR, Zotti MJ, Smagghe G, Christiaens O. 2016. RNAi Efficiency, Systemic Properties, and

434 Novel Delivery Methods for Pest Insect Control: What We Know So Far. Front Physiol 7:553

435 DOI10.3389/fphys.2016.00553

436 Khajuria C, Vélez AM, Rangasamy M, Wang H, Fishilevich E, Frey ML, Carneiro NP, Gandra P,

437 Narva KE, Siegfried BD. 2015. Parental RNA interference of genes involved in embryonic 438 development of the western corn rootworm, Diabrotica virgifera virgifera LeConte. Insect 
439 Biochem Mol Biol 63:54-62 DOI 10.1016/j.ibmb.2015

440 Kurdyumov AS, Manuvera VA, Baskova IP, Lazarev VN. 2015. A comparison of the enzymatic

441 properties of three recombinant isoforms of thrombolytic and antibacterial protein--Destabilase-

442 Lysozyme from medicinal leech. BMC Biochem 16:27 DOI: 10.1186/s12858-015-0056-3.Li J,

443 Ma L, Lin Z, Zou Z, Lu Z. 2016. Serpin-5 regulates prophenoloxidase activation and antimicrobial

444 peptide pathways in the silkworm, Bombyx mori. Insect Biochem Mol Biol. 73:27-37 DOI

445 10.1016/j.ibmb.2016.04.003

446 Mao J, Zeng F. 2014. Plant-mediated RNAi of a gap gene-enhanced tobacco tolerance against the 447 Myzus persicae. Transgenic Res 23(1):145-52 DOI 10.1007/s11248-013-9739-y

448 Meng FL, Ran RX, Li Y, Li N, Li HZ, Wang ZK, Li WB. 2017a. RNAi-mediated knockdown of 449 a serine protease gene (Spbtry1) from SPB (soybean pod borer) affects the growth and mortality 450 of the pest. Florida Entomologist 100(3):607-615 DOI /10.1653/024.100.0319.

451 Meng F, Li Y, Zang Z, Li N, Xue R, Cao Y, Li T, Zhou Q, Li W. 2017b. Expression of the Double452 stranded RNA of the soybean pod borer Leguminivora glycinivorella (Lepidoptera: Tortricidae) 453 Ribosomal Protein P0 Gene Enhances the Resistance of Transgenic Soybean Plants. Pest Manag 454 Sci. (12):2447-2455 DOI 10.1002/ps.4637

455 Meng FL, Yang MY, Li Y, Li TY,Liu XX, Wang GY, Wang ZC, Jin XH, Li WB. 2017c. 456 Functional analysis of RNA interference-related soybean pod borer (Lepidoptera) genes based on 457 transcriptome sequences. Front. Physiol. DOI 10.3389/fphys.2018.00383

458 Monwan W, Amparyup P, Tassanakajon A. 2017. A snake-like serine proteinase (PmSnake) 459 activates prophenoloxidase-activating system in black tiger shrimp Penaeus monodon. Dev Comp 460 Immunol. 67:229-238 DOI 10.1016/j.dci.2016.09.016. 
461 Myllymäki H, Valanne S, Rämet M. 2014. The Drosophila imd signaling pathway. J Immunol. 462 192(8):3455-62 DOI 10.4049/jimmunol.1303309

463 Parsons B, Foley E. 2016. Cellular immune defenses of Drosophila melanogaster. Dev Comp 464 Immunol 58:95-101 DOI 10.1016/j.dci.2015.12.019

465 Rao XJ, Zhan MY, Pan YM, Liu S, Yang PJ, Yang LL, Yu XQ. 2017. Immune functions of insect 466 BGRPs and their potential application. Dev Comp Immunol. pii: S0145-305X(17)30524-4 DOI 467 10.1016/j.dci.2017.12.007

468 Roh KB, Kim CH, Lee H, Kwon HM, Park JW, Ryu JH, Kurokawa K, Ha NC, Lee WJ, Lemaitre 469 B, Söderhäll K, Lee BL. 2009. Proteolytic cascade for the activation of the insect toll pathway 470 induced by the fungal cell wall component. J Biol Chem. 284(29):19474-81 DOI 471 10.1074/jbc.M109.007419

472 Shukla JN, Kalsi M, Sethi A, Narva KE, Fishilevich E, Singh S, Mogilicherla K, Palli SR. 2016.

473 Reduced stability and intracellular transport of dsRNA contribute to poor RNAi response in 474 lepidopteran insects. RNA Biol 13(7):656-69 DOI 10.1080/15476286.2016.1191728

475 Song LW, Wen XY, Zang LS, Ruan CC, Shi SS, Shao XW, Zhang F. 2015. Parasitism and 476 Suitability of Different Egg Ages of the Leguminivora glycinivorella (Lepidoptera: Tortricidae)

477 for Three Indigenous Trichogramma Species. J Econ Entomol 108(3):933-9 DOI $478 \quad 10.1093 /$ jee/tov027

479 Sievers F, Wilm A, Dineen D, Gibson TJ, Karplus K, Li W, Lopez R, McWilliam H, Remmert M, 480 Söding J, Thompson JD, Higgins DG. 2011. Fast, scalable generation of high-quality protein 481 multiple sequence alignments using Clustal Omega. Mol Syst Biol. 7:539. doi: $482 \quad 10.1038 / \mathrm{msb} .2011 .75$. 
483 Takeda K, Akira S. 2004. Microbial recognition by Toll-like receptors. J Dermatol Sci. 34(2):73-

48482 DOI 10.1016/j.jdermsci.2003.10.002

485 Tamura K, Stecher G, Peterson D, Filipski A, Kumar S. 2011. MEGA5: molecular evolutionary 486 genetics analysis using maximum likelihood, evolutionary distance, and maximum parsimony 487 methods. Mol Biol Evol 28(10):2731-9 DOI 10.1093/molbev/msr121

488 Terenius O, Papanicolaou A, Garbutt JS, Eleftherianos I, Huvenne H, Kanginakudru S, 489 Albrechtsen M, An C, Aymeric JL, Barthel A, Bebas P, Bitra K, Bravo A, Chevalier F, Collinge 490 DP, Crava CM, de Maagd RA, Duvic B, Erlandson M, Faye I, Felföldi G, Fujiwara H, Futahashi 491 R, Gandhe AS, Gatehouse HS, Gatehouse LN, Giebultowicz JM, Gómez I, Grimmelikhuijzen CJ, 492 Groot AT, Hauser F, Heckel DG, Hegedus DD, Hrycaj S, Huang L, Hull JJ, Iatrou K, Iga M, 493 Kanost MR, Kotwica J, Li C, Li J, Liu J, Lundmark M, Matsumoto S, Meyering-Vos M, Millichap 494 PJ, Monteiro A, Mrinal N, Niimi T, Nowara D, Ohnishi A, Oostra V, Ozaki K, Papakonstantinou 495 M, Popadic A, Rajam MV, Saenko S, Simpson RM, Soberón M, Strand MR, Tomita S, Toprak U, 496 Wang P, Wee CW, Whyard S, Zhang W, Nagaraju J, Ffrench-Constant RH, Herrero S, Gordon K, 497 Swevers L, Smagghe G. 2011. RNA interference in Lepidoptera: an overview of successful and 498 unsuccessful studies and implications for experimental design. J Insect Physiol 57(2):231-45 DOI 499 10.1016/j.jinsphys.2010.11.006.

500 Troll JV, Adin DM, Wier AM, Paquette N, Silverman N, Goldman WE, Stadermann FJ, Stabb 501 EV, McFall-Ngai MJ. 2009. Peptidoglycan induces loss of a nuclear peptidoglycan recognition 502 protein during host tissue development in a beneficial animal-bacterial symbiosis. Cell Microbiol 503 11(7):1114-27 DOI 10.1111/j.1462-5822.2009.01315.x

504 Ulrich J, Dao VA, Majumdar U, Schmitt-Engel C, Schwirz J, Schultheis D, Ströhlein N, 
505 Troelenberg N, Grossmann D, Richter T, Dönitz J, Gerischer L, Leboulle G, Vilcinskas A, Stanke

506 M, Bucher G. 2015. Large scale RNAi screen in Tribolium reveals novel target genes for pest

507 control and the proteasome as prime target. BMC Genomics 16:674 DOI 10.1186/s12864-015-

508 1880-y.

509 Valanne S, Wang JH, Rämet M. 2011. The Drosophila Toll signaling pathway. J Immunol

510 186(2):649-56 DOI: 10.4049/jimmunol.1303309

511 Wang L, Yang S, Han L, Fan D, Zhao K. 2014. Phenotypic plasticity of HSP70s gene expression

512 during diapause: signs of evolutionary responses to cold stress among Soybean Pod Borer

513 populations (Leguminivora glycinivorella) in Northeast of China. PLoS One 9(10):e109465 (2014)

514 DOI 10.1371/journal.pone.0109465

515 Wang J, Aksoy S. 2012. PGRP-LB is a maternally transmitted immune milk protein that influences

516 symbiosis and parasitism in tsetse's offspring. Proc Natl Acad Sci U S A. 109(26):10552-7

517 DOI10.1073/pnas.1116431109

518 Wu C, Chen C, Dai J, Zhang F, Chen Y, Li W, Pastor-Pareja JC, Xue L. 2015. Toll pathway

519 modulates TNF-induced JNK-dependent cell death in Drosophila. Open Biol 5(7):140171 DOI:

$520 \quad 10.1098 /$ rsob. 140171

521 Xu Q, Lu A, Xiao G, Yang B, Zhang J, Li X, Guan J, Shao Q, Beerntsen BT, Zhang P, Wang C,

522 Ling E. 2012. Transcriptional profiling of midgut immunity response and degeneration in the

523 wandering silkworm, Bombyx mori. PLoS One 7(8):e43769 DOI 10.1371/journal.pone.0043769

524 Xue, Q.G., Schey, K.L., Volety, A.K., Chu, F.L., La Peyre, J.F. 2004. Purification and 525 characterization of lysozyme from plasma of the eastern oyster (Crassostrea

526 virginica). Comp Biochem Physiol B Biochem Mol Biol 139(1):11-25 DOI: 
527 10.1016/j.cbpc.2004.05.011

528 Yang PJ, Zhan MY, Ye C, Yu XQ, Rao XJ. 2017. Molecular cloning and characterization of a

529 short peptidoglycan recognition protein from silkworm Bombyx mori. Insect Mol Biol. 26(6):665-

530676 DOI $10.1111 /$ imb. 12330

531 Zaidman-Rémy A1, Hervé M, Poidevin M, Pili-Floury S, Kim MS, Blanot D, Oh BH, Ueda R,

532 Mengin-Lecreulx D, Lemaitre B. 2006. The Drosophila amidase PGRP-LB modulates the immune

533 response to bacterial infection. Immunity 24(4):463-73 DOI 10.1016/j.immuni.2006.02.012

534 Yang J, Wang X, Tang S, Shen Z, Wu J. 2015. Peptidoglycan recognition protein S2 from

535 silkworm integument: characterization, microbe-induced expression, and involvement in the

536 immune-deficiency pathway. J Insect Sci 15. pii: 20 DOI 10.1093/jisesa/iev007

537 Zhao GY, Wang J, Han YP, Teng WL, Sun GL, Li WB. 2008. Identification of QTL underlying

538 the resistance of soybean to pod borer, Leguminivora glycinivorella (Mats.) obraztsov, and

539 correlations with plant, pod and seed traits. Euphytica 164: 275-282 DOI 1016/S2095-3119

540 Zhou J, Zhao S, Fang WH, Zhou JF, Zhang JX, Ma H, Lan JF, Li XC. 2017. Newly identified

541 invertebrate-type lysozyme (Splys-i) in mud crab (Scylla paramamosain) exhibiting muramidase-

542 deficient antimicrobial activity. Dev Comp Immunol 74:154-166 DOI: 10.1016/j.dci.2017.04.017 
Figure 1

Phylogenetic relationships among PGRPs from Leguminivora glycinivorella and Drosophila melanogaster

The phylogenetic tree was constructed using MEGA5.0 with a neighbour-joining approach. The bootstrap percentages (1,000 replicates) are provided next to the branches. The first two letters of each PGRP name indicate the species (Dm, D. melanogaster; Lg, L. glycinivorella). 


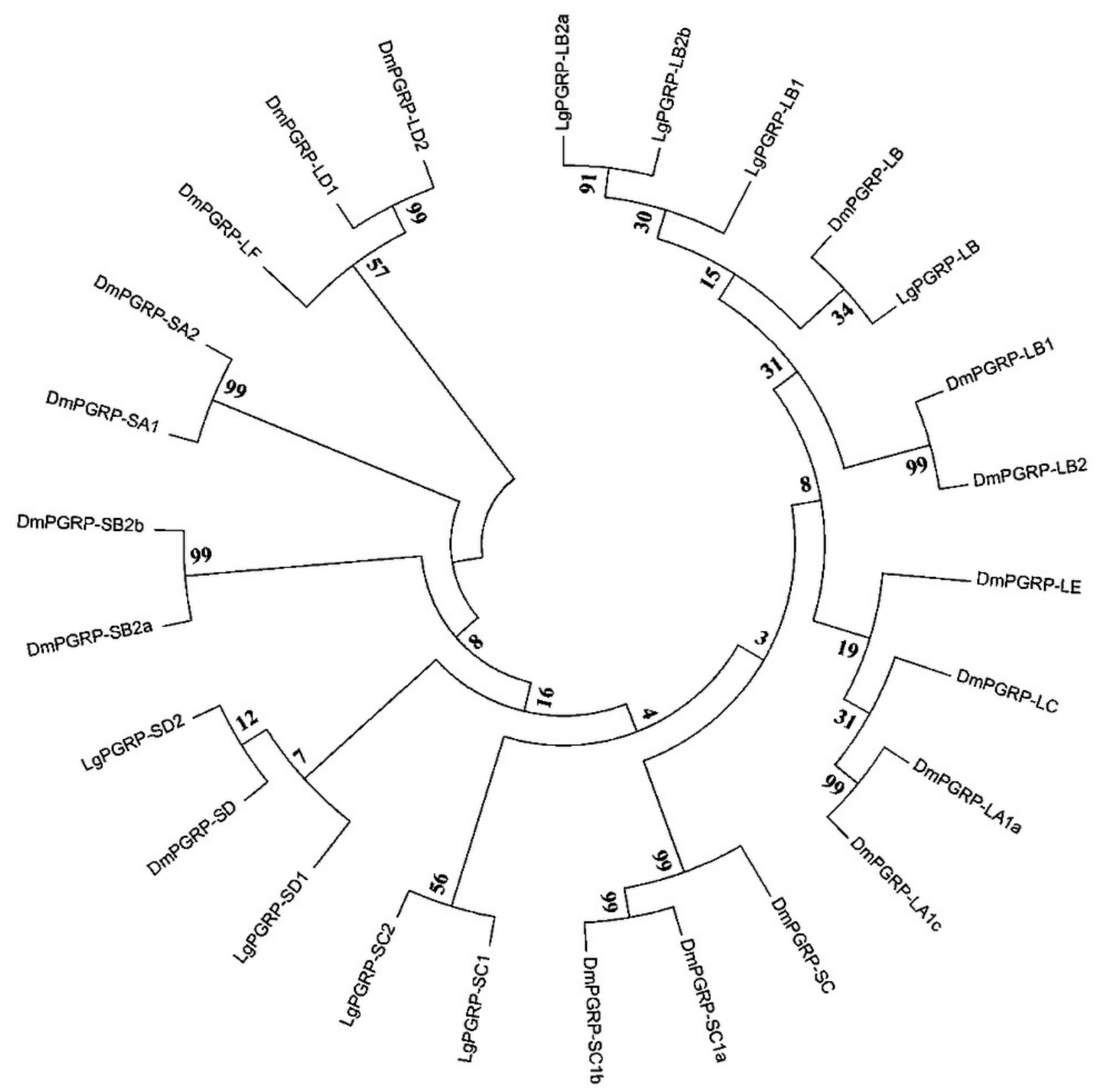




\section{Figure 2}

The analysis of Toll receptors of theL. glycinivorella

A) Phylogenetic relationships among Toll receptors from Leguminivora glycinivorella,Bombyx mori and Drosophila melanogaster. The phylogenetic tree was constructed using MEGA5.0 with a neighbour-joining approach. $L g, L$. glycinivorella; Bm,Bombyx mori; $D m, D$. melanogaster; TLR, Toll like receptor. (B) Predicted domains of the L.glycinivorellaToll receptors. The domain organization were predicted using the SMART program (http://smart.embl.de/). The extracellular leucine-rich repeats (LRRs), rectangles; LRR Cterminal domain, small ellipses; intracytoplasmic TIR domains, big ellipses; signal peptides, red rectangles; transmembrane domain, blue bar.

A

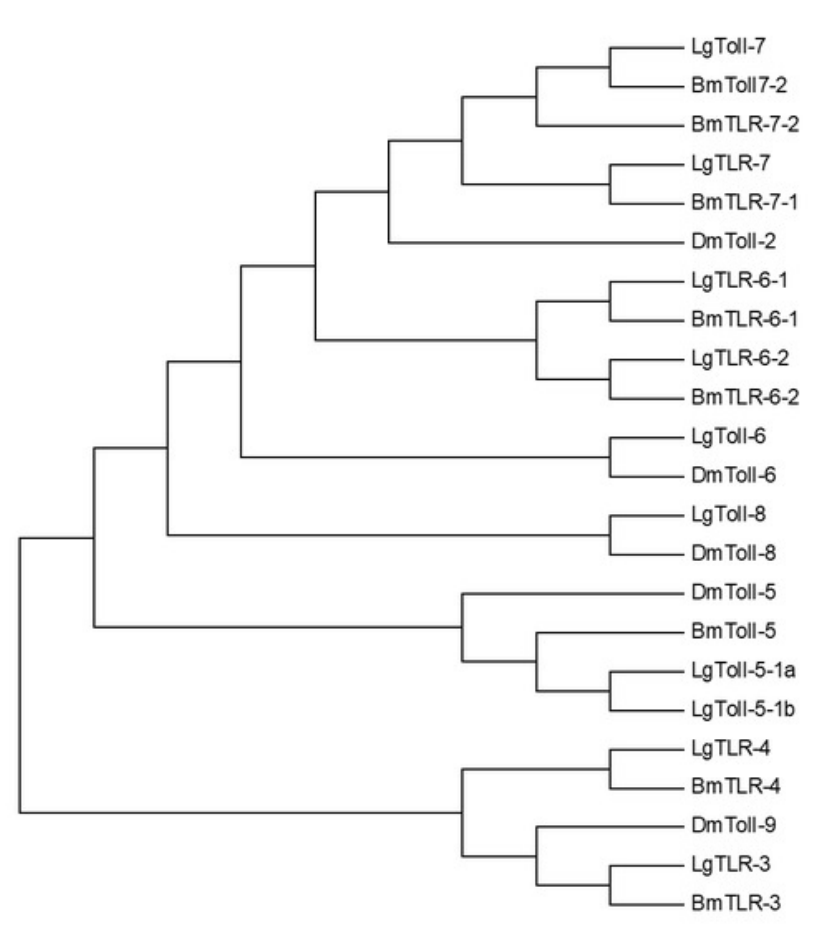

B

LgTLR-3

LgTLR-4

LgToll-5-1a

LgToll-5-1b

LgToll-6

LgTLR-6-1

LgTLR-6-2

LgToll-7

LgTLR-7

LgToll-8
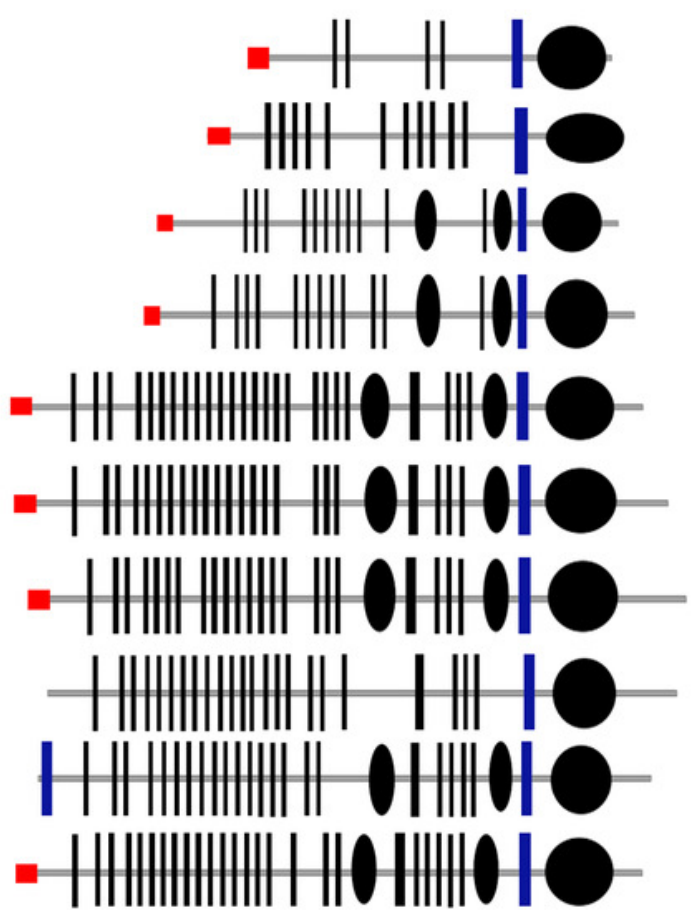
Figure 3

Mortality rates of soybean pod borer larvae fed an artificial diets supplemented separately with dsRNA (10 $\mu \mathrm{g} / \mathrm{g}$ ) for 11 candidate RNA interference target genes.

Columns represent mean $\pm S E$. Different letters above the column indicate significant difference $(p<0.0009)$.

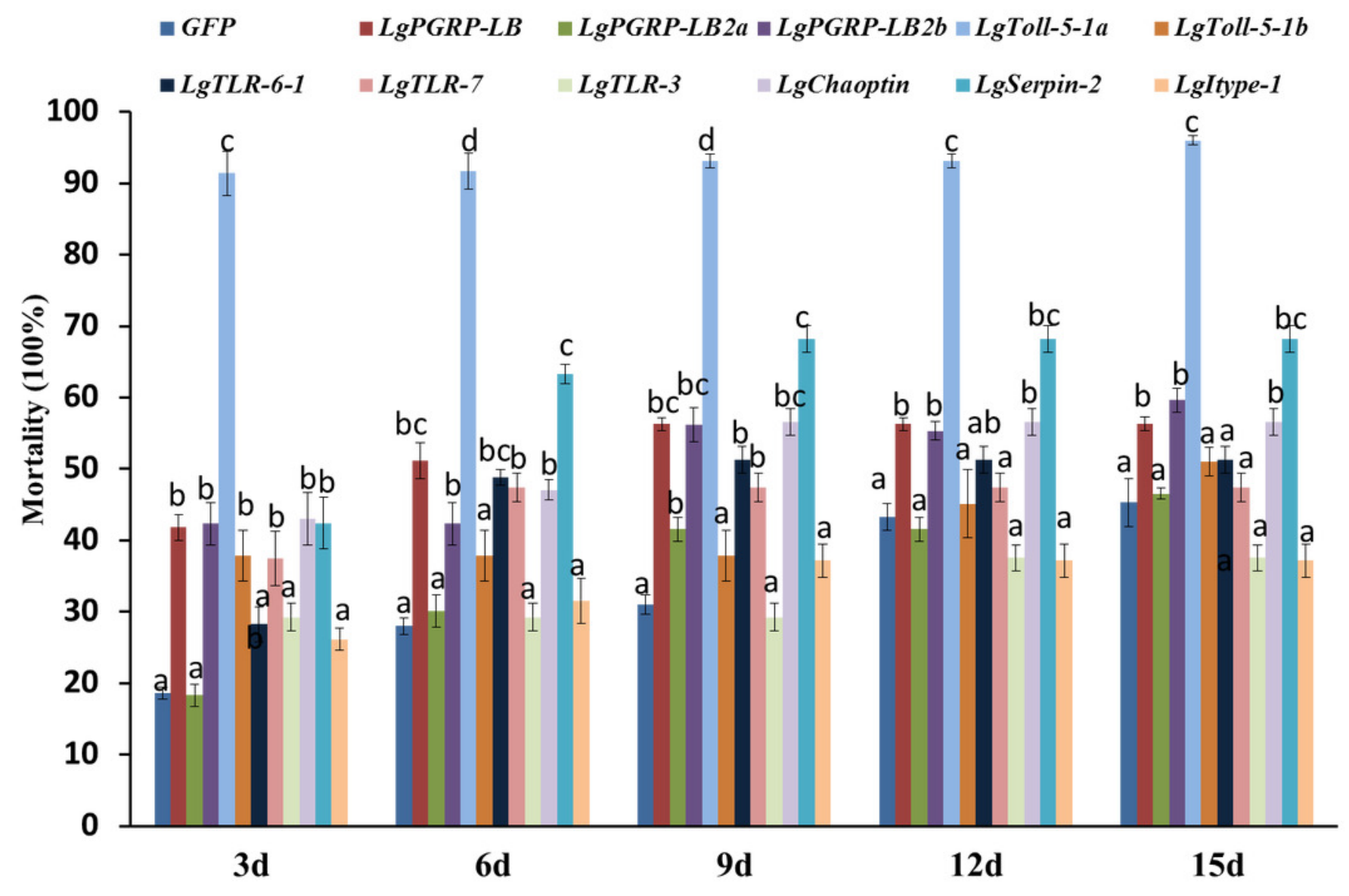


Figure 4

Body weights of soybean pod borer larvae fed artificial diets supplemented separately with dsRNA $(10 \mu \mathrm{g} / \mathrm{g})$ for 11 candidate RNA interference target genes.

Columns represent mean $\pm S E$. Different letters above the column indicate significant difference $(p<0.0009)$.

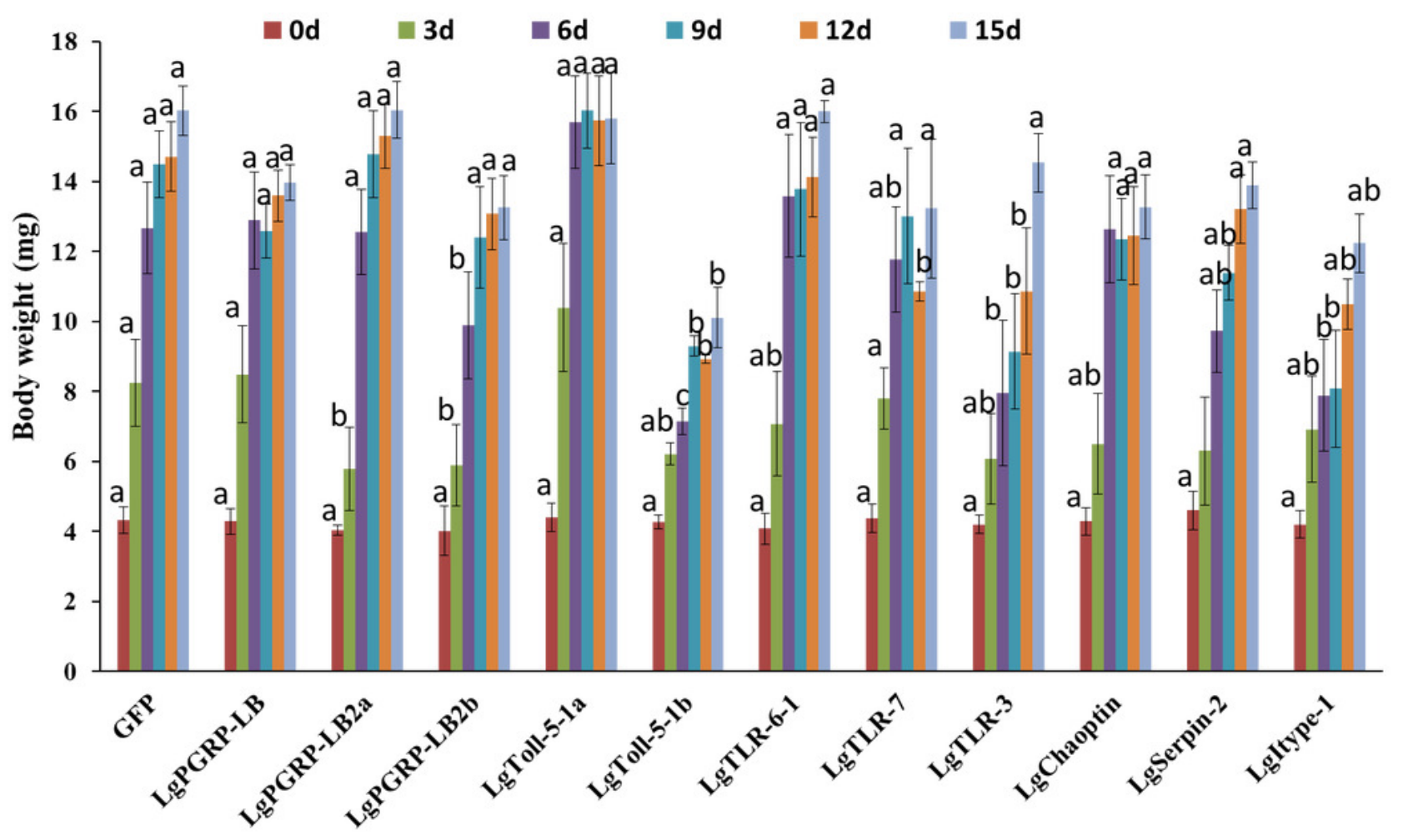




\section{Figure 5}

Images of soybean pod borer larvae fed an artificial diet supplemented separately with dsRNA $(10 \mu \mathrm{g} / \mathrm{g})$ for 11 candidate RNA interference target genes for $15 \mathrm{~d}$.

A) The larvae fed an artificial diet containing dsRNA for LgPGRP-LB. (B) The larvae fed an artificial diet containing dsRNA for LgPGRP-LB2a. (C) The larvae fed an artificial diet containing dsRNA for LgPGRP-LB2b. (D) The larvae fed an artificial diet containing dsRNA for LgToll-5-1a, only one survived after $15 \mathrm{~d}$. (E) The larvae fed an artificial diet containing dsRNA for LgToll-5-1b. (F) The larvae fed an artificial diet containing dsRNA for LgTLR-6-1b. (G) The larvae fed an artificial diet containing dsRNA for LgTLR-7. (H) The larvae fed an artificial diet containing dsRNA for LgTLR-3. (I)The larvae fed an artificial diet containing dsRNA for LgChaoptin. (J) The larvae fed an artificial diet containing dsRNA for LgSerpin-2. (K) The larvae fed an artificial diet containing dsRNA for Lgltype-1. (L) The larvae fed an artificial diet containing dsRNA for GFP.

$\mathbf{A}$

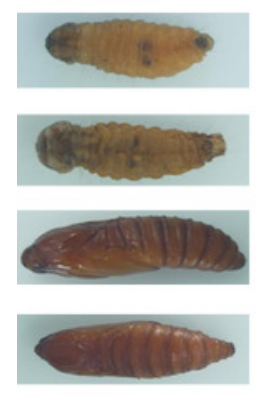

G

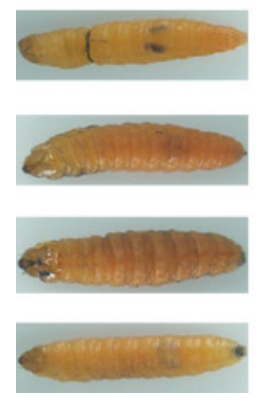

B

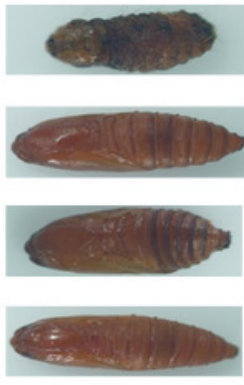

H

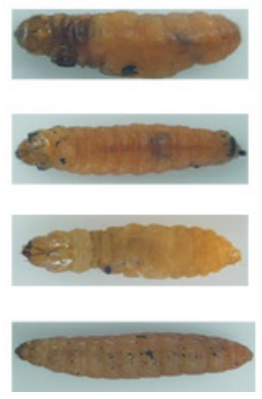

C

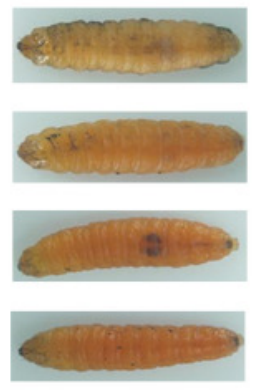

I

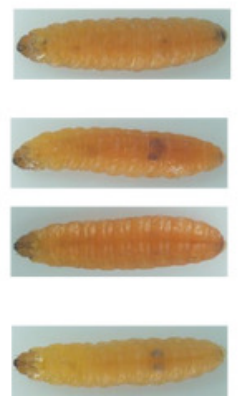

D

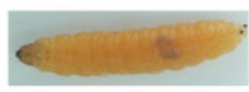

$\mathbf{J}$

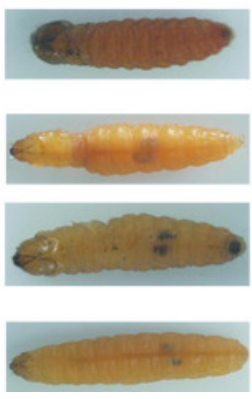

E

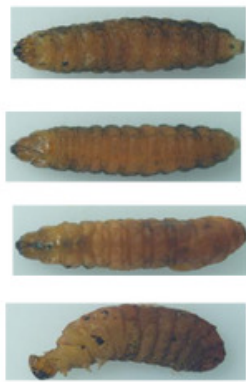

K

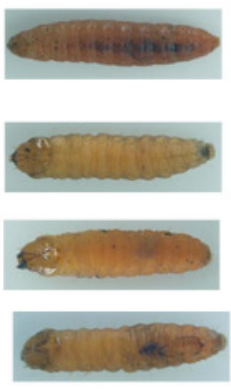

F

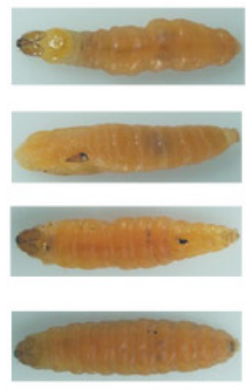

L

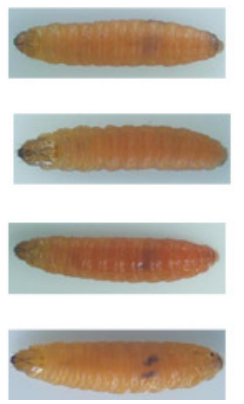


Figure 6

Relative expression levels of 11 candidate RNA interference target genes at different time points after soybean pod borer larvae were fed separate artificial diets containing the respective dsRNA $(10 \mu \mathrm{g} / \mathrm{g})$.

Quantitative real-time polymerase chain reactions were completed using total RNA extracted from surviving larvae. Columns represent mean \pm SE. Different letters above the column indicate significant difference $(p<0.002)$

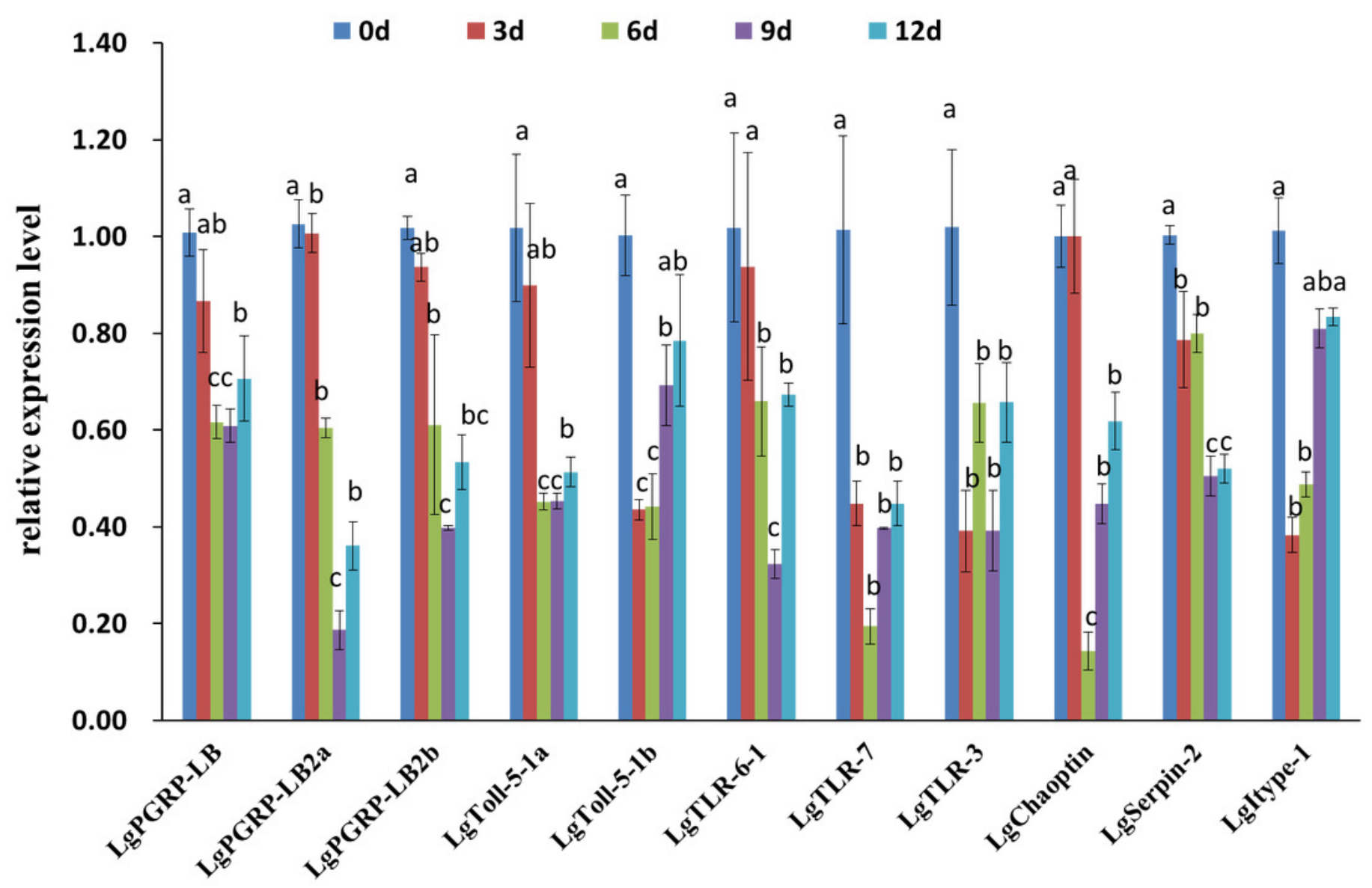

